Präv Gesundheitsf 2021 · 16:188-192 https://doi.org/10.1007/s11553-021-00836-x Eingegangen: 16 . November 2020

Angenommen: 5. Februar 2021

Online publiziert: 5. März 2021

(c) Der/die Autor(en) 2021

\author{
Alexander Behnke' ${ }^{1}\left[\right.$ Roberto Rojas $^{2}(\mathbb{D}) \cdot$ Anne Gärtner $^{3} \mathbb{D}$ \\ ${ }^{1}$ Klinische \& Biologische Psychologie, Institut für Psychologie und Pädagogik, Universität Ulm, Ulm, \\ Deutschland \\ ${ }_{2}^{2}$ Psychotherapeutische Hochschulambulanz, Institut für Psychologie und Pädagogik, Universität Ulm, \\ Ulm, Deutschland \\ ${ }^{3}$ Differentielle und Persönlichkeitspsychologie, Fakultät Psychologie, Technische Universität Dresden, \\ Dresden, Deutschland
}

\title{
Emotionsregulation im Rettungsdienst
}

\section{Zusammenhänge mit beruflichem Stress, Belastungssymptomatik und Arbeitszufriedenheit von Beschäftigten im Rettungsdienst}

\section{Hintergrund}

\section{Risikoberuf Rettungsdienst}

Rettungsdienstmitarbeiter ${ }^{*}$ innen (RDM) werden im Rahmen ihrer Tätigkeit wiederholt mit (potenziell) traumatischen Ereignissen konfrontiert und erleben chronischen tätigkeitsbedingten Stress $[4,15]$. Aufgrund seiner besonderen emotionalen Stressbelastung gehört der medizinische Rettungsdienst zu den Berufen mit dem höchsten Risiko für Traumafolgestörungen wie Posttraumatische Belastungsstörung (PTBS, 10-14,6\%), Depression (15\%) und Angststörungen (15\%), Alkoholkonsumstörung und erhöhte Suizidalität [20, 27]. Vielfach weisen Studien über Erkrankungshäufigkeiten in Risikoberufen jedoch methodische Schwächen auf, wie das Fehlen geeigneter Kontrollgruppen [20, 27]. Neben der hohen physischen Beanspruchung trägt die emotionale Belastung der RDM wesentlich $\mathrm{zu}$ einem hohen Krankenstand, starker Personalfluktuation und erhöhten Raten krankheitsbedingter Arbeitsunfähigkeit und Frühverrentung bei $[25,26]$. Der individuelle Stil der RDM, negative und aversive Gefühle etwa infolge traumatischer Einsatzerlebnisse zu bewältigen, kann entscheidend dazu beitragen, inwieweit RDM ihrer tä- tigkeitsbedingten Belastung standhalten und spielt eine kritische Rolle für den Erhalt ihrer Gesundheit, Lebensqualität und Arbeitsfähigkeit [6, 17].

\section{Forschungsfeld Emotions- regulation}

Emotionsregulation bezeichnet alle Prozesse, mithilfe derer eine Person beeinflusst, welche Emotionen sie empfindet, wann sie diese Gefühle hat, wie sie sie erlebt und ausdrückt [1]. „Adaptive“ Emotionsregulationsstrategien werden mit persönlichem Wohlbefinden sowie psychischer und körperlicher Gesundheit in Verbindung gebracht, während „maladaptive“ Strategien mit einer höheren Anfälligkeit für Stress und die Entwicklung psychischer und körperlicher Belastungssymptome einhergehen [1]. $\mathrm{Zu}$ den maladaptiven Strategien gehören (a) anhaltendes Grübeln über das Erlebte (Rumination), (b) der Versuch Gefühle $z u$ unterdrücken (Unterdrücken), und (c) der Versuch, Gedanken an das Erlebte zu vermeiden (Vermeiden). $\mathrm{Zu}$ den adaptiven Strategien gehören Versuche, (a) Hindernisse planvoll zu überwinden (Problemlösen), (b) positive Sichtweisen auf belastende Erlebnisse zu entwickeln (Neubewerten) sowie (c) auch unange- nehme Gefühle zuzulassen, zu tolerieren und anzunehmen (Akzeptanz) [1].

\section{Fragestellung und Studienziele}

Wir untersuchten im Rahmen einer Querschnittsstudie [11], (a) inwieweit RDM die Emotionsregulationsstrategien Neubewerten, Akzeptanz, Problemlösen, Grübeln, Unterdrücken und Vermeiden gewohnheitsmäßig anwenden, um mit emotionalem Stress umzugehen. Zudem untersuchten wir (b) den Zusammenhang der Strategienutzung mit dem beruflichen Stresserleben, der depressiven und posttraumatischen Stresssymptomatik, körperlichen Belastungssymptomen (u.a. Schmerzen, gastrointestinale Beschwerden, Kreislaufprobleme) sowie der Arbeitszufriedenheit der RDM. Eine ausführliche Beschreibung der verwendeten Fragebogen findet sich bei Gärtner et al. [11].

\section{Stichprobe}

Insgesamt nahmen 102 Beschäftigte zweier südwestdeutscher Rettungswachen an der Studie teil [11]. Die Teilnehmenden waren überwiegend männlich (65\%), zwischen 18 und 61 (Medi$a n=27,5)$ Jahren alt und hatten zwischen einer Woche und 35 (Median=3,3) Jah- 


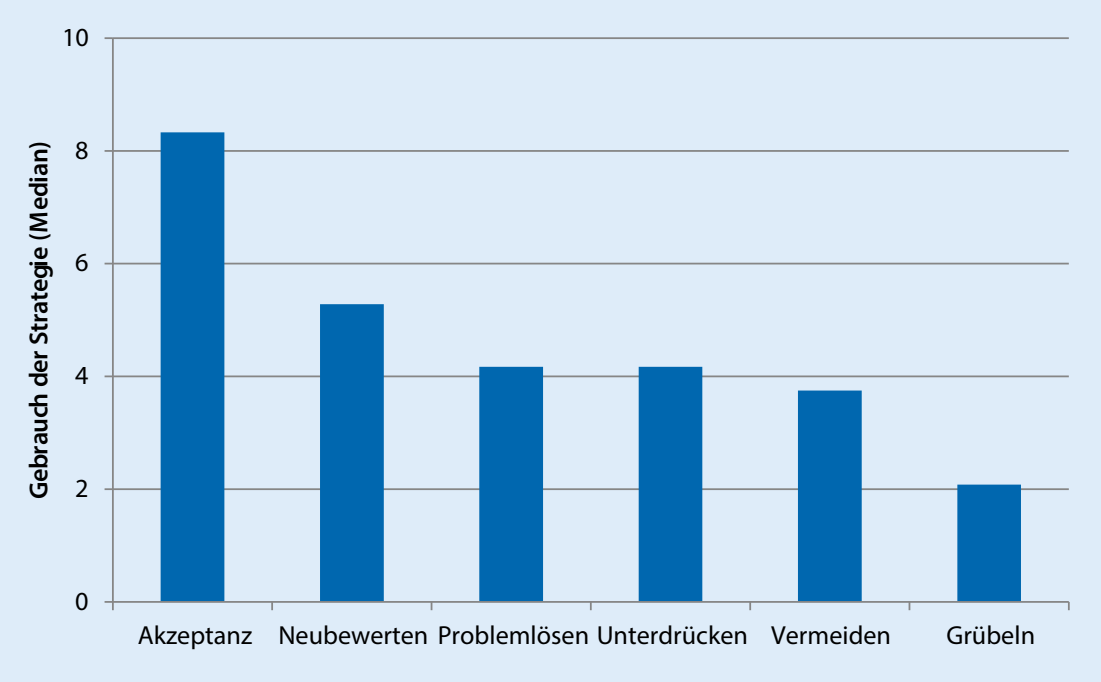

Abb. 1 ॥ Gewohnheitsmäßiger Gebrauch von Emotionsregulationsstrategien im Rettungsdienst

ren Berufserfahrung. Die Mehrzahl $(60 \%)$ der Teilnehmenden hatte eine Ausbildung zum Notfallsanitäter absolviert; $32 \%$ arbeiteten ehrenamtlich im Rettungsdienst. Die Stichprobe korrespondierte zufriedenstellend mit der Gesamtzahl der Beschäftigten $(n=318)$ im Hinblick auf Alter, Geschlecht, Berufserfahrung und Arbeitsort [11].

\section{Ergebnisse und Diskussion}

\section{Nutzung und Zusammenhänge von Emotionsregulations- strategien im Rettungsdienst}

In unserer Studie nutzten RDM häufiger adaptive als maladaptive Strategien (• Abb. 1). Die Beschäftigten gaben an, am häufigsten die Strategien Akzeptanz und Neubewerten anzuwenden, gefolgt von Problemlösen, Unterdrücken und Vermeiden; vergleichsweise selten grübelten sie über negative Erlebnisse und Gefühle [11].

\section{Maladaptive Strategien}

Obwohl maladaptive Strategien seltener genutzt wurden als adaptive Strategien, zeigten sie die stärksten Zusammenhänge mit erhöhter Belastungssymptomatik: die häufigere Nutzung von Unterdrücken, Grübeln und Vermeiden ging mit erhöhtem beruflichen Stresserleben und stärkeren depressiven, posttraumati- schen und körperlichen Belastungssymptomen einher (• Tab. 1). Dieses Ergebnis reiht sich ein in die Befunde einer Vielzahl von Untersuchungen, in welchen maladaptive Emotionsregulationsstrategien konsistente Zusammenhänge mit negativen Belastungsfolgen zeigten. Dies zeigt sich nicht zuletzt auch darin, dass die Arbeitszufriedenheit bei den RDM geringer war, die häufiger grübelten und Gefühle unterdrückten (•Tab. 1).

\section{Problemlösen und Neubewerten}

Im Hinblick auf die adaptiven Strategien ergab sich ein heterogenes Bild: So gingen die Strategien Problemlösen und Neubewerten weder mit geringerem Stresserleben, noch geringeren Belastungssymptomen oder einer höheren Arbeitszufriedenheit einher (•Tab. 1). Auch vorherige Studien ergaben inkonsistente Ergebnisse hinsichtlich der Nützlichkeit dieser Strategien im Rettungsdienst $[8,9,24]$. Die Ergebnisse im Rettungsdienst stehen somit in deutlichem Kontrast zu den positiven Effekten der Strategien Problemlösen und Neubewerten in der Allgemeinbevölkerung oder bei Patienten mit psychischen Störungen [1]. Eine mögliche Erklärung könnte sein, dass die Nützlichkeit von Emotionsregulationsstrategien stark kontextabhängig ist $[6,17]$ : So erscheinen die Bildung neuer Perspektiven auf negative Erlebnisse sowie ein geplantes
Vorgehen geeignet zur Bewältigung von alltäglichen und zwischenmenschlichen Problemen. Im Unterschied dazu sind RDM im Rahmen ihrer Einsätze mit Extremsituationen konfrontiert [4], auf die sie sich nicht planvoll vorbereiten können, da sie deren Umstände nicht kontrollieren können (z.B. mangelnde Technik zum Befreien eingeklemmter Patienten). Weiterhin können sie das Erlebte (z.B. plötzlichen Kindstod) kaum positiv umdeuten [2]. Vielmehr ist $\mathrm{zu}$ vermuten, dass Versuche einer nachträglichen Umdeutung bzw. planvollen Veränderung unkontrollierbarer Umstände die Neigung zum Grübeln begünstigen, was die Entwicklung psychopathologischer Symptome eher beschleunigen denn bremsen könnte $[11,18]$.

\section{Akzeptanz als geeignete Strategie}

In unserer Studie ging der gewohnheitsmäßige Gebrauch von Akzeptanz mit geringeren psychischen und körperlichen Belastungssymptomen einher. Allerdings zeigten sich keine Vorteile im Hinblick auf das berufliche Stresserleben und die Arbeitszufriedenheit der RDM (•Tab. 1); d.h. auch wenn Akzeptanz keine Vorteile im Hinblick auf das Stresserleben und die Arbeitszufriedenheit zu bieten scheint, könnte die Bereitschaft auch unangenehme Gefühle anzunehmen langfristig zum Schutz der psychischen und körperlichen Gesundheit beitragen. Tatsächlich identifizierten auch vorherige Studien die Neigung zur Akzeptanz unangenehmer Gefühle als potenziellen Schutzfaktor für die Gesundheit im Rettungsdienst und anderen traumaexponierten Berufen [10, 23, 29, 30]. Akzeptanz bezeichnet eine beobachtende, nicht wertende Beziehung zu den eigenen Gefühlen, Gedanken und Überzeugungen. Emotionale Akzep$\tan z$ ist ein zentrales Prinzip achtsamkeitsbasierter Therapieansätze (z.B. der "acceptance and commitment therapy" [12]), welche die Überwindung starrer Überzeugungs-, Bewertungs- oder Bewältigungsmuster anstreben, um die psychologische Flexibilität für die Anpassung an schwierige Lebenssituationen zu schaffen. Akzeptanz erlaubt eine selbstdistanzierte Perspektive auf belas- 
Präv Gesundheitsf 2021 · 16:188-192 https://doi.org/10.1007/s11553-021-00836-x

(c) Der/die Autor(en) 2021

A. Behnke · R. Rojas · A. Gärtner

\section{Emotionsregulation im Rettungsdienst. Zusammenhänge mit beruflichem Stress, Belastungssymptomatik und Arbeitszufriedenheit von Beschäftigten im Rettungsdienst}

\section{Zusammenfassung}

Hintergrund. Rettungsdienstmitarbeiter*innen (RDM) sind wiederholt mit traumatischen Einsatzerlebnissen und chronischem arbeitsbedingtem Stress konfrontiert. Um dieser Belastung standzuhalten, ist die Bewältigung von emotionalem Stress entscheidend. Allerdings ist kaum bekannt, welche Strategien RDM zur Regulation unangenehmer Emotionen einsetzen und wie sich diese Strategien auf das Wohlbefinden und die Gesundheit der RDM auswirken. Ziel der Arbeit. Wir untersuchten, inwieweit RDM die Emotionsregulationsstrategien Neubewerten, Akzeptanz, Problemlösen, Grübeln (Rumination), Unterdrücken und Vermeiden anwenden und welche Zusammenhänge diese Strategien mit dem
Stresserleben, der Arbeitszufriedenheit und der Belastungssymptomatik der RDM zeigen. Material und Methoden. In einer Querschnittserhebung schätzten 102 RDM zweier südwestdeutscher Rettungswachen anhand standardisierter Fragebogen ihren Emotionsregulationsstil, ihr berufliches Stresserleben, ihre Arbeitszufriedenheit und ihre depressiven, posttraumatischen und körperlichen Belastungssymptome ein. Ergebnisse. Korrelationsanalysen ergaben, dass die Neigung zu häufigem Grübeln, Vermeiden und Unterdrücken mit höherem Stresserleben, stärkeren Belastungssymptomen und geringerer Arbeitszufriedenheit einhergeht. Die Akzeptanz unangenehmer Gefühle war mit geringeren Belastungssym- ptomen assoziiert, während Neubewerten und Problemlösen keine Korrelation mit dem Stresserleben, der Zufriedenheit und der Belastungssymptomatik der RDM zeigten. Diskussion. Unsere Ergebnisse bestätigen die dysfunktionale Rolle von Grübeln, Vermeiden und Unterdrücken für das Wohlbefinden und die Gesundheit in Risikoberufen. Die kontextspezifische Wirksamkeit von Emotionsregulationsstrategien sollte bei der Planung zielgruppenspezifischer Präventionsmaßnahmen stärker berücksichtigt werden.

Schlüsselwörter

Berufliche Traumatisierung · Stressbewältigung · Akzeptanz · Stress · Arbeitszufriedenheit

\section{Emotion regulation in the Emergency Medical Services. Association with the personnel's occupational stress, stress symptomatology, and job satisfaction}

\section{Abstract}

Background. Emergency medical services (EMS) personnel are recurrently exposed to traumatic mission incidents and chronic work-related stress. In order to withstand this strain, coping with emotional stress is crucial. However, little is known about the strategies EMS personnel use to regulate their unpleasant emotions, and how these strategies affect the personnel's well-being and health.

Objectives. We investigated the extent to which EMS personnel use emotion-regulation strategies (i.e. reappraisal, acceptance, problem solving, rumination, suppression, and avoidance), and how these strategies relate to the EMS personnel's stress experience, job satisfaction, and stress symptoms.

\begin{abstract}
Materials and methods. In a cross-sectional survey, 102 EMS personnel of two southwestern German ambulance stations reported on standardised questionnaires their use of emotion-regulation strategies, perceived work-related stress, job satisfaction, as well as depressive, posttraumatic, and physical stress symptoms.

Results. Correlation analyses showed that the tendency to engage frequently in rumination, avoidance, and suppression was associated with higher perceived stress, more severe stress symptoms, and lower job satisfaction. Acceptance of unpleasant feelings was associated with less severe stress symptoms, while reappraisal and problem solving showed no correlation with the EMS personnel's
\end{abstract}

stress perception, job satisfaction, and stress symptoms.

Conclusions. Our results confirm the dysfunctional role of rumination, avoidance, and suppression for the well-being and health in at-risk professions. The context-specific adaptiveness of emotion-regulation strategies should be considered when developing profession-specific health-prevention measures.

\section{Keywords}

Occupational trauma $\cdot$ Stress coping . Acceptance $\cdot$ Occupational stress $\cdot$ Job satisfaction tende Erfahrungen, vermeidet negative selbstbezogene Emotionen wie Scham oder Schuld, verhindert den Einstieg in einen schädlichen Grübelkreislauf und kann so der Entstehung von Traumafolgesymptomatik entgegenwirken [3, 21].

Zusammenfassend zeigen unsere Ergebnisse, dass RDM eher adaptive als maladaptive Emotionsregulationsstrategien anwenden. Unter den untersuchten
Strategien schien ausschließlich Akzep$\operatorname{tanz}$ eine geeignete Strategie für RDM zu sein, während Neubewerten und Problemlösen für Beschäftigte des medizinischen Rettungsdienstes keine Vorteile bieten könnten. Die Neigung zu maladaptiver Emotionsregulation, d.h. Grübeln, Vermeiden und Unterdrücken, ging mit einer Verstärkung des wahrgenommenen arbeitsbedingten Stresses, der psychischen und körperlichen Belastungs- symptomatik sowie einer geringeren Arbeitszufriedenheit bei RDM einher.

\section{Schlussfolgerungen für die be- rufliche Gesundheitsförderung}

Ein Baustein der primärpräventiven $\mathrm{Ge}$ sundheitsförderung in Rettungsdienst und anderen traumaexponierten Berufen (u. a. in Polizei, Feuerwehr, Katastrophenhilfe, Hilfsorganisationen, Militär) 
Tab. 1 Korrelationen der Emotionsregulationsstrategien mit Wohlbefinden und Gesundheit.

(Aus [11])

\begin{tabular}{|c|c|c|c|c|c|}
\hline & $\begin{array}{l}\text { Wahrge- } \\
\text { nommener } \\
\text { Stress }\end{array}$ & $\begin{array}{l}\text { Posttrau- } \\
\text { matische } \\
\text { Symptome }\end{array}$ & $\begin{array}{l}\text { Depressive } \\
\text { Symptome }\end{array}$ & $\begin{array}{l}\text { Körperliche Belas- } \\
\text { tungssymptome }\end{array}$ & $\begin{array}{l}\text { Arbeits- } \\
\text { zufrieden- } \\
\text { heit }\end{array}$ \\
\hline Akzeptanz & $-0,09$ & $-0,21^{*}$ & $-0,25^{*}$ & $-0,29^{* *}$ & 0,03 \\
\hline Neubewerten & $-0,05$ & $-0,05$ & $-0,03$ & $-0,05$ & 0,17 \\
\hline Problemlösen & 0,16 & 0,19 & 0,12 & 0,08 & $-0,16$ \\
\hline Vermeiden & $-0,01$ & $0,23^{*}$ & $0,26^{* *}$ & $0,24^{*}$ & $-0,02$ \\
\hline Unterdrücken & $0,24^{*}$ & $0,44^{* * *}$ & $0,48^{* * *}$ & $0,41^{* * *}$ & $-0,30^{* *}$ \\
\hline Grübeln & $0,32^{* *}$ & $0,51^{* * *}$ & $0,67^{* * *}$ & $0,60^{* * *}$ & $-0,33^{* * *}$ \\
\hline
\end{tabular}

kann darin bestehen, Beschäftigten Fertigkeiten $\mathrm{zu}$ vermitteln, mithilfe derer sie besser mit den emotionalen Beanspruchungen ihrer Tätigkeit umgehen können. Die Ergebnisse unserer Studie sowie vorheriger Studien [10, 23, 29, 30] deuten darauf hin, dass Gesundheitspräventionsmaßnahmen im Rettungsdienst (a) eine Reduzierung der Neigung zum Grübeln, Vermeiden und Unterdrücken von Gefühlen anstreben sowie (b) einen akzeptanzbasierten Umgang mit aversiven Gefühlen bei RDM und andere traumaexponierten Beschäftigten fördern sollten. In ersten Studien bei Beschäftigten von Feuerwehr, Polizei und Militär zeigten sog. „Mindfulness-based stress regulation-“ (MBSR-)Trainings positive Auswirkungen auf verschiedene Stressindikatoren wie selbstberichteten Ärger, wahrgenommenen Stress, psychische und körperliche Belastungssymptome sowie verschiedene physiologische Stressmarker [13, 14, 16]. Darüber hinaus legen Metaanalysen nahe, dass MBSR-Trainings in moderatem Maße zur Reduktion der Stress- und Angstsymptome bei Ärzt*innen beitragen [22] und vorteilhafte Effekte auf physiologische Stressmarker wie Blutdruck, Herzfrequenz sowie die Konzentration zirkulierender Stresshormone und Entzündungsmarker haben [19]. Daneben können MBSR-Trainings dazu beitragen, die Neigung zu maladaptiver Emotionsregulation, wie das Grübeln, zu reduzieren [21].

Für den erfolgreichen Umgang mit emotionalem Stress ist es jedoch nicht hinreichend, dass Beschäftigte möglichst oft und möglichst viele adaptive Regulationsstrategien einsetzen. Vielmehr bedarf die effektive Bewältigung unangenehmer Gefühle des Wissens, welche Strategien für welche konkrete Situation geeignet sind, sowie der Fähigkeit, zwischen geeigneten Strategien flexibel wechseln $\mathrm{zu}$ können [6, 17]. Demnach könnten RDM besonders von Trainings profitieren, die (a) ihr individuelles Repertoire adaptiver Strategien erweitern und (b) den flexiblen Wechsel zwischen Strategien einüben [6, 17]. Ein Beispiel für ein solches repertoire- und flexibilitätsorientiertes Training ist das Training emotionaler Kompetenzen (TEK) [5]. In zwölf 90-minütigen Sitzungen werden sieben Kompetenzen der adaptiven Emotionsregulation vermittelt: die Fähigkeit, (i) Emotionen bewusst wahrzunehmen, (ii) Emotionen zu identifizieren und korrekt zu benennen, (iii) zu erkennen, was Emotionen verursacht und aufrechterhält, (iv) Emotionen aktiv und adaptiv zu modifizieren, (v) negative Emotionen zu akzeptieren und $\mathrm{zu}$ tolerieren, (vi) Situationen zu konfrontieren, die wahrscheinlich unerwünschte Emotionen auslösen, und (vii) in belastenden Situationen effektive Selbsthilfe zu leisten. Buruck und Dörfel adaptierten das TEK für den Rettungsdienstkontext und führten eine Pilotstudie zur Wirksamkeit des Trainings mit 107 Auszubildenden des Rettungsdienstes durch [7]. Das TEK reduzierte die emotionale Erschöpfung und den Zynismus in der Versuchsgruppe im Vergleich zur Kontrollgruppe. Die Vorteile des Trainings waren zurückzuführen auf Zugewinne der Kompetenzbereiche Annehmen, Tolerieren und Regulieren negativer Emotionen sowie eine erhöhte Bereitschaft, sich mit unangenehmen Gefühlen auseinanderzusetzen [7].
Trotz der vielversprechenden kurzund mittelfristigen Auswirkungen kompetenzorientierter Trainings wie TEK bedarf es der weiteren Erforschung ihrer langfristigen Wirksamkeit. Zu diesem Ergebnis kommen auch Wild et al. [28] in einem aktuellen Überblick zu gesundheitsförderlichen Präventionsansätzen im Rettungsdienst und deren empirischer Wirksamkeitsprüfung: Demnach existieren vielversprechende Ansätze für Stressresilienztrainings, deren abschließende Wirksamkeitsprüfung jedoch überwiegend noch aussteht. Keinen wirksamen Beitrag zum Schutz von Gesundheit und Wohlbefinden von RDM liefern Einstellungsuntersuchungen (d.h. die Auswahl vermeintlich „resilienter“ Bewerber) sowie reine Psychoedukation. Hingegen existieren Belege über die förderliche Wirkung realistischer Einsatztrainings sowie Maßnahmen zur Sensibilisierung von Führungskräften und der Etablierung eines sozial unterstützenden kollegialen bzw. betrieblichen Klimas [28].

\section{Fazit für die Praxis}

- Rettungsdienstmitarbeiter*innen, die stärker zu maladaptiver Emotionsregulation (u.a. Grübeln, Gefühle unterdrücken, Vermeiden) neigen, empfinden mehr beruflichen Stress, zeigen stärkere psychische und körperliche Belastungssymptome und geringere Arbeitszufriedenheit.

- Die Akzeptanz von unangenehmen Gefühlen geht mit geringeren psychischen und körperlichen Belastungssymptomen bei Rettungsdienstmitarbeiter*innen einher.

- Die gemeinhin als adaptiv geltenden Emotionsregulationsstrategien Problemlösen und Neubewerten scheinen aufgrund der spezifischen Anforderungen des Rettungsberufes wenig adaptiv für Rettungsdienstmitarbeiter*innen zu sein.

- Verhaltensorientierte Prävention sollte Bewältigungsstrategien fördern, welche für den spezifischen Berufskontext adaptiv sind (z. B. achtsamkeitsbasierte Stressregulation), sowie das Repertoire und die Flexibilität der Beschäftigten im Einsatz adaptiver Strategien erhöhen. 


\section{Korrespondenzadresse}

Alexander Behnke, Dipl.-Psych.

Klinische \& Biologische Psychologie, Institut für Psychologie und Pädagogik, Universität Ulm Albert-Einstein-Allee 47, 89081 Ulm,

Deutschland

alexander.behnke@uni-ulm.de

\section{Dr. Anne Gärtner, Dipl.-Psych.}

Differentielle und Persönlichkeitspsychologie, Fakultät Psychologie, Technische Universität Dresden

01069 Dresden, Deutschland anne_gaertner@tu-dresden.de

Funding. Open Access funding enabled and organized by Projekt DEAL.

\section{Einhaltung ethischer Richtlinien}

Interessenkonflikt. A. Behnke, R. Rojas und A. Gärtner geben an, dass kein Interessenkonflikt besteht.

Die Studie wurde durch die Ethikkommission der Universität Ulm geprüft und für zulässig befunden (Ethikvotum Nr. 269/16).

Open Access. Dieser Artikel wird unter der Creative Commons Namensnennung 4.0 International Lizenz veröffentlicht, welche die Nutzung, Vervielfältigung Bearbeitung, Verbreitung und Wiedergabe in jeglichem Medium und Format erlaubt, sofern Sie den/die ursprünglichen Autor(en) und die Quelle ordnungsgemäß nennen, einen Link zur Creative Commons Lizenz beifügen und angeben, ob Änderungen vorgenommen wurden.

Die in diesem Artikel enthaltenen Bilder und sonstiges Drittmaterial unterliegen ebenfalls der genannten Creative Commons Lizenz, sofern sich aus der Abbildungslegende nichts anderes ergibt. Sofern das betreffende Material nicht unter der genannten Creative Commons Lizenz steht und die betreffende Handlung nicht nach gesetzlichen Vorschriften erlaubt ist, ist für die oben aufgeführten Weiterverwendungen des Materials die Einwilligung des jeweiligen Rechteinhabers einzuholen.

Weitere Details zur Lizenz entnehmen Sie bitte der Lizenzinformation auf http://creativecommons.org/ licenses/by/4.0/deed.de.

\section{Literatur}

1. Aldao A, Nolen-Hoeksema S, Schweizer S (2010) Emotion-regulation strategies across psychopathology: a meta-analytic review. Clin Psychol Rev 30:217-237. https://doi.org/10.1016/j.cpr.2009. 11.004

2. Avraham N, Goldblatt H, Yafe E (2014) Paramedics' experiences and coping strategies when encountering critical incidents. Qual Health Res 24:194-208. https://doi.org/10.1177/ 1049732313519867

3. Ayduk Ö, Kross E (2010) Analyzing negative experiences without ruminating: the role of selfdistancing in enabling adaptive self-reflection. Soc
Personal Psychol Compass 4:841-854. https://doi. org/10.1111/j.1751-9004.2010.00301.x

4. Behnke A, Rojas R, Karrasch S, Hitzler M, Kolassa I-T (2019) Deconstructing traumatic mission experiences: identifying critical incidents and their relevance for the mental and physical health among emergency medical service personnel. Front Psychol 10:2305. https://doi.org/10.3389/ fpsyg.2019.02305

5. Berking $M$ (2011) Training emotionaler Kompetenzen.Springer, Berlin, Heidelberg

6. Bonanno GA, Burton CL (2013) Regulatory flexibility: an individual differences perspective on coping and emotion regulation. Perspect Psychol Sci 8:591-612. https://doi.org/10.1177/ 1745691613504116

7. Buruck G, Dörfel D (2018) Emotionsregulation in der Arbeit am Beispiel Rettungsdienst. Initiative Gesundheit und Arbeit, Dresden

8. Cicognani E, Pietrantoni L, Palestini L, Prati G (2009) Emergency workers' quality of life: the protective role of sense of community, efficacy beliefs and coping strategies. Soc Indic Res 94:449-463. https://doi.org/10.1007/s11205-009-9441-x

9. Clohessy S, Ehlers A (1999) PTSD symptoms, response to intrusive memories and coping in ambulance service workers. Br J Clin Psychol 38:251-265. https://doi.org/10.1348/014466599162836

10. Counson I, Hosemans D, Lal TJ, Mott B, Harvey SB, Joyce $S$ (2019) Mental health and mindfulness amongst Australian fire fighters. BMC Psychol 7:34. https://doi.org/10.1186/s40359-019-0311-2

11. Gärtner A, Behnke A, Conrad D, Kolassa I-T, Rojas R (2019) Emotion regulation in rescue workers: differential relationship with perceived workrelated stress and stress-related symptoms. Front Psychol 9:2744. https://doi.org/10.3389/fpsyg. 2018.02744

12. Hayes SC, Luoma JB, Bond FW, Masuda A, Lillis J (2006) Acceptance and commitment therapy: model, processes and outcomes. Behav Res Ther 44:1-25. https://doi.org/10.1016/j.brat.2005.06. 006

13. Johnson DC, Thom NJ, Stanley EA, Haase L, Simmons AN, Shih PB, Thompson WK, Potterat EG Minor TR, Paulus MP (2014) Modifying resilience mechanisms in at-risk individuals: a controlled study of mindfulness training in marines preparing for deployment. Am J Psychiatry 171:844-853. https://doi.org/10.1176/appi.ajp.2014.13040502

14. Kaplan JB, Bergman AL, Christopher M, Bowen S, Hunsinger $M$ (2017) Role of resilience in mindfulness training for first responders. Mindfulness 8:1373-1380. https://doi.org/10.1007/s12671017-0713-2

15. Karutz H, Overhagen M, Stum J (2013) Psychische Belastungen im Wachalltag von Rettungsdienstmitarbeitern und Feuerwehrleuten. Präv Gesundförd 8:204-211. https://doi.org/10.1007/s11553012-0373-y

16. Khoury B, Sharma M, Rush SE, Fournier C (2015) Mindfulness-based stress reduction for healthy individuals: a meta-analysis. J Psychosom Res 78:519-528. https://doi.org/10.1016/j.jpsychores. 2015.03.009

17. Levy-Gigi E, Bonanno GA, Shapiro AR, RichterLevin G, Kéri S, Sheppes G (2015) Emotion regulatory flexibility sheds light on the elusive relationship between repeated traumatic exposure and posttraumatic stress disorder symptoms. Clin Psychol Sci 4:28-39. https://doi.org/10.1177/ 2167702615577783

18. Marmar CR, McCaslin SE, Metzler TJ, Best $S$, Weiss DS, Fagan J, Liberman A, Pole N, Otte C,
Yehuda R, Mohr D, Neylan T (2006) Predictors of posttraumatic stress in police and other first responders. Ann N Y Acad Sci 1071:1-18. https:// doi.org/10.1196/annals.1364.001

19. Pascoe MC, Thompson DR, Jenkins ZM, Ski CF (2017) Mindfulness mediates the physiological markers of stress: systematic review and metaanalysis. J Psychiatr Res 95:156-178. https://doi org/10.1016/j.jpsychires.2017.08.004

20. Petereit-Haack G, Bolm-Audorff U, Romero Starke K, Seidler A (2020) Occupational risk for post-traumatic stress disorder and traumarelated depression: a systematic review with metaanalysis. Int J Environ Res Public Health 17:9369. https://doi.org/10.3390/ijerph17249369

21. Querstret D, Cropley M (2013) Assessing treatments used to reduce rumination and/or worry: a systematic review. Clin Psychol Rev 33:996-1009. https://doi.org/10.1016/j.cpr.2013.08.004

22. Regehr C, Glancy D, Pitts A, LeBlanc VR (2014) Interventions to reduce the consequences of stress in physicians: a review and meta-analysis. J Nerv Ment Dis 202:353-359. https://doi.org/10.1097/ NMD. 0000000000000130

23. Setti I, Argentero P (2014) The role of mindfulness in protecting firefighters from psychosomatic malaise. Traumatology 20:134-141. https://doi. org/10.1037/h0099398

24. Shepherd L, Wild J (2014) Emotion regulation, physiological arousal and PTSD symptoms in trauma-exposed individuals. J Behav Ther Exp Psychiatry 45:360-367. https://doi.org/10.1016/j. jbtep.2014.03.002

25. Sterud T, Ekeberg $\varnothing$, Hem E (2006) Health status in the ambulance services: a systematic review. BMC Health Serv Res 6:82. https://doi.org/10.1186/ 1472-6963-6-82

26. Suyama J, Rittenberger JC, Patterson PD, Hostler D (2009) Comparison of public safety provider injury rates. Prehosp Emerg Care 13:451-455. https://doi. org/10.1080/10903120903144908

27. Wagner SL, White N, Regehr C, White M, Alden LE, Buys N, Carey MG, Corneil W, Fyfe T, Matthews LR, Randall C, Krutop E, Fraess-Phillips A (2020) Ambulance personnel: systematic review of mental health symptoms. Traumatology. https:// doi.org/10.1037/trm0000251

28. Wild J, Greenberg N, Moulds ML, Sharp M-L, Fear N, Harvey S, Wessely S, Bryant RA (2020) Pre-incident training to build resilience in first responders: recommendations on what to and what not to do. Psychiatry 83:128-142. https://doi.org/10.1080/ 00332747.2020.1750215

29. Williams V, Ciarrochi J, Deane FP (2010) On being mindful, emotionally aware, and more resilient: longitudinal pilot study of police recruits. Aust Psychol 45:274-282. https://doi.org/10.1080/ 00050060903573197

30. Zhao Y, An Y, Sun X, Liu J (2019) Self-acceptance, post-traumatic stress disorder, post-traumatic growth, and the role of social support in Chinese rescue workers. J Loss Trauma 25:264-277. https:// doi.org/10.1080/15325024.2019.1672935 